\title{
latrogenic AKI and Agranulocytosis: a Cautious Tale of Medication Use in Elderly Patients
}

\author{
Meng Qin\#, Zhen Cang\#, Yingli Liu* and Bo Shen
}

Department of Nephrology, Shanghai Ninth People's Hospital, Shanghai Jiao Tong University School of Medicine, Shanghai 200011, China

\#These authors contribute equally to this work and are listed as co-first author.

*Corresponding author: Yingli Liu, Division of Nephrology, Shanghai Ninth People's Hospital, Shanghai Jiao Tong University School of Medicine, Shanghai 200011, China, Tel: +86 18616375719; Fax: +86-21-64085875; E-mail: 18616375719@163.com

Received date: March 28, 2017; Accepted date: April 20, 2017; Published date: April 25, 2017

Citation: Meng Q, Zhen C, Yingli L, Bo S (2017) latrogenic AKI and Agranulocytosis: A Cautious tale of Medication use in Elderly Patients. J Clin Exp Nephrol 1: 33. doi: 10.21767/2472-5056.100033

Copyright: (C) 2017 Yingli L, et al. This is an open-access article distributed under the terms of the Creative Commons Attribution License, which permits unrestricted use, distribution, and reproduction in any medium, provided the original author and source are credited.

\section{Abstract}

A 83-year-old female patient suffered from acute kidney injury a month after percutaneous coronary intervention. Two weeks before hospitalization, she experienced nausea and intermittent watery diarrhea. Laboratory test showed high levels of serum creatine kinase and lactate dehydrogenase, which were remarkably decreased after ceasing rosuvastatin and valsartan treatment. Despite the recovery of renal function, unfortunately one week later, she was diagnosed with agranulocytosis because of antibiotic therapy. When we stopped meropenem and switched to granulocyto-colony stimulating factor treatment, her white blood cell count returned to a normal range. In our case, both rhabdomyolysis and agranulocytosis were induced by drugs. We provided a literature review of the causes of acute kidney injury and the pathogenesis of rhabdomyolysis and agranulocytosis. We discussed how drugs might lead to these complications. This case highlights the need to be aware of the potential side effects of drugs, especially those given to elderly patients who are likely to be receiving several medications concurrently for managing chronic diseases.

\section{Keywords:}

Acute kidney injury; Rhabdomyolysis;

Agranulocytosis

\section{Introduction}

Due to the increase in life expectancy, more geriatric patients are present in the hospital. They often have several chronic diseases and are taking numerous medicines at the same time. Drug induced acute kidney injury is very common, especially for the contrast-induced nephropathy after percutaneous coronary intervention and other interventional operations. Here, we report a case of acute kidney injury induced by rhabdomyolysis in an elderly patient who recently underwent a percutaneous coronary intervention. She also suffered from agranulocytosis induced by antibiotic drugs. We provide a literature review of the causes of acute kidney injury and the pathogenesis of rhabdomyolysis and agranulocytosis. We hope that this case would raise the awareness of considering the potential side effects of drugs especially in geriatric patients. Many older adults have multiple medical conditions, such as hypertension, arthritis, heart disease, cancer, and diabetes mellitus, which require multiple medications for proper treatment [1]. A national survey found that $57 \%$ of US women aged $\geq 65$ years took $\geq 5$ prescription medications and $12 \%$ took $\geq 10$ medications [2].

\section{Case History}

A 83-year-old female patient was admitted due to a continuous increase in serum creatinine $(\mathrm{sCr})$ level peaked at $715 \mu \mathrm{mol} / \mathrm{L}$. She has a past medical history of coronary heart disease and atrial fibrillation for over 10 years, taking amiodarone and betaloc to control ventricular rate. She also has a history of high blood pressure (maximum 180/100 $\mathrm{mmHg}$ ) over 20 years, which is managed by valsartan $80 \mathrm{mg} / \mathrm{d}$, furosemide $20 \mathrm{mg} / \mathrm{d}$, and spironolactone $20 \mathrm{mg} / \mathrm{d}$. One month before admission, this patient had undergone a coronary angiography and percutaneous coronary intervention (PCI) after presenting with chest tightness and chest pain. She routinely took clopidogrel $75 \mathrm{mg} / \mathrm{d}$ and aspirin $100 \mathrm{mg} / \mathrm{d}$ for preventing platelet aggregation and rosuvastatin $10 \mathrm{mg} / \mathrm{d}$ for plaque stabilization after PCl. Her sCr increased from $122 \mu \mathrm{mol} / \mathrm{L}$ to 138 $\mu \mathrm{mol} / \mathrm{L}$ two days after operation. Two weeks later, she suffered from nausea and intermittent watery diarrhea. At the same time, she had soy urine without fever and muscle pain or swelling.

On the first day of hospitalization, her blood pressure was 90/61 $\mathrm{mmHg}$; heart rate was 108 per minute with atrial fibrillation. Physical examination showed that her abdomen was flat, with no tenderness and rebound tenderness. No edema was observed in her lower limb. Laboratory blood test and urinalysis reported a sCr of $489 \mathrm{umol} / \mathrm{L}$, serum $\mathrm{K}+$ of 2.98 $\mathrm{mmol} / \mathrm{L}$, serum $\mathrm{Ca}^{2+}$ of $2.04 \mathrm{mmol} / \mathrm{L}$, serum creatine kinase (CK) of $4621 \mathrm{U} / \mathrm{L}$, serum lactate dehydrogenase (LDH) of $764 \mathrm{U} / \mathrm{L}$, white blood cell (WBC) count of $5.4 \times 10^{9} / \mathrm{L}$, neutrophil percentage (N\%) of $83.4 \%$, urine UBG $(+/-)$, and urine ERY $(+)$. 
Abdominal ultrasonography revealed an enlargement of the gallbladder, cholecystitis, multiple gallbladder stones, and bile duct dilatation. Both kidneys were of normal size, but their structure appeared fuzzy.

In hospital, her blood pressure had once dropped to 50/30 $\mathrm{mmHg}$, and her urine volume was only $200 \mathrm{~mL}$ in the first $24 \mathrm{~h}$. We, therefore, administered colloidal solution (Albumin, Intralipid) to expand the blood volume and dopamine to increase the blood pressure. We stopped using rosuvastatin and valsartan. In addition, we started administering meropenem 1.0 $\mathrm{g}$ bid by intravenous infusion to treat potential infection, Caltrate $D$ to regulate calcium phosphorus metabolism, potassium chloride to manage hypokalemia, and sodium bicarbonate to alkalize urine. On the third day after admission, this patient entered a polyuria stage with her urine volume went up to 3.8 L/day, whereas the CK and LDH levels decreased remarkably (Figure 1). Her renal function also recovered gradually (Figure 2 ).

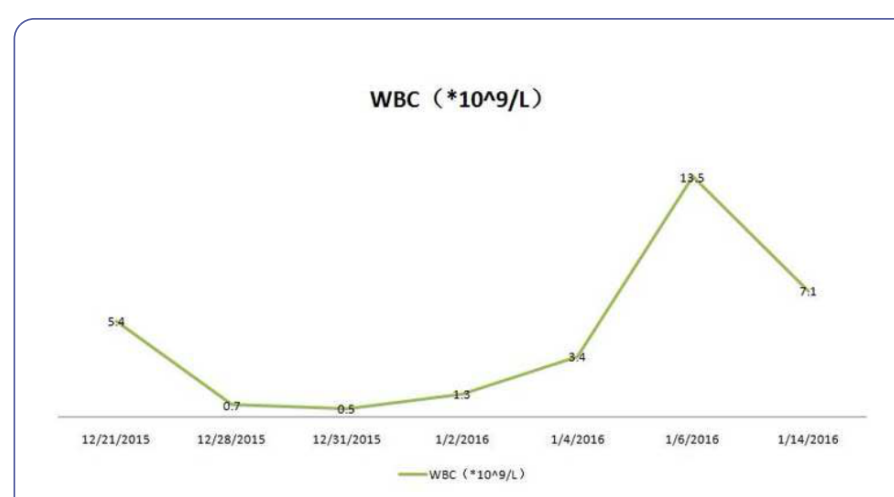

Figure 1: CK and LDH levels decreased remarkably.

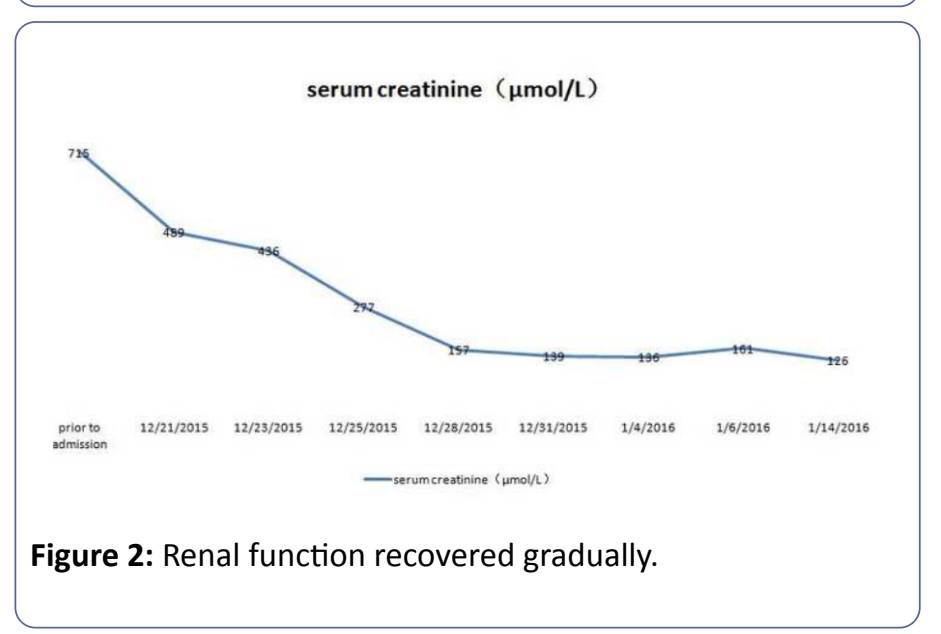

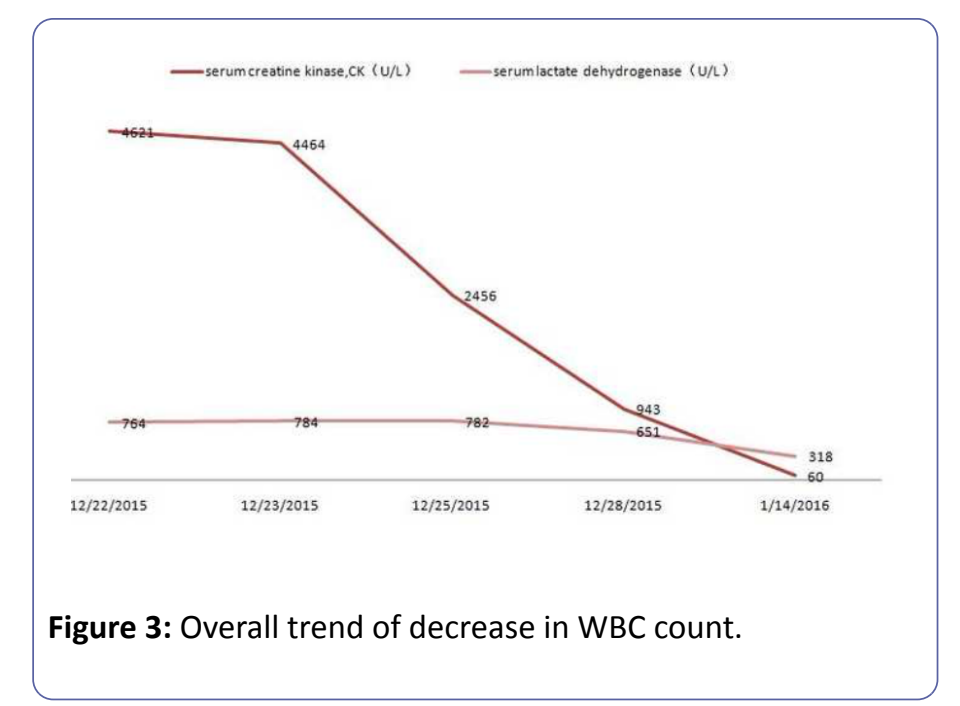

One week after admission, her blood report showed an overall trend of decrease in WBC count (Figure 3), indicative of an agranulocytosis that might be induced by meropenem intravenous infusion. Therefore, we immediately stopped meropenem and switched to granulocyto-colony stimulating factor (G-CSF) $100 \mathrm{u} / \mathrm{d}$ by hypodermic injection. One week later, her WBC count returned to a normal range.

\section{Discussion}

According to the Acute Kidney Injury Network (AKIN) [3] and Risk, Injury, Failure, Loss, and End-stage (RIFLE) kidney disease criteria [4], this patient can be diagnosed as stage 3 acute kidney injuries (AKI). We considered it is an acute exacerbation of chronic kidney disease on the basis of her preoperative $\mathrm{sCr}$, fuzzy kidney structure, and the signs of hypocalcemia, hyperphosphatemia and hyperparathyroidism. The potential causes of such acute exacerbation include (1) contrast-induced nephropathy (CIN) after the $\mathrm{PCl},(2)$ application of RAS inhibitors, (3) hypovolemia and prerenal renal failure caused by acute gastrointestinal infections and diarrhea, and (4) rhabdomyolysis caused by statins. AKI affects an estimated $13-18 \%$ of inpatients $[5,6]$. A study shows that the morbidity rate for AKI is $21.6 \%$ in adults and $33.7 \%$ in children, whereas the mortality rates for adults and children are $23.9 \%$ and $13.8 \%$, respectively [5]. The mortality rate is even as high as $60 \%$ for elderly patients in the intensive care unit, $80 \%$ of whom requires a renal replacement therapy [7]. The most common causes of AKI are infection, renal ischemia, and use of nephrotoxic drugs $[8,9]$. The risk for AKI is determined by patient-specific factors (e.g. advanced age, chronic kidney disease and other preexisting chronic medical conditions) and other predisposing factors (e.g. sepsis and surgery) [10]. AKI is strongly associated with advanced age, and the recent increase in AKI incidence is linked to the growing size of the elderly population. With the emergence of an aging society and advances in medical care, $\mathrm{PCl}$ is widely performed in elderly patients with coronary heart disease to improve their survival rate. Unfortunately, the administration of contrast media (CM) may result in $\mathrm{CIN}$, which is the third most common cause of AKI in patients admitted to hospital [11], subsequently leading to a prolonged hospital stay and increased mortality [12]. According to the European Society of Urogenital Radiology 
(ESUR), CIN is defined as an increase of $\mathrm{sCr}$ level by more than $44 \mu \mathrm{mol} / \mathrm{L}(0.5 \mathrm{mg} / \mathrm{dL})$ or $25 \%$ over baseline within 3 days following intravascular CM exposure [13]. In this reported case, the preoperative $\mathrm{sCr}$ of this patient was $122 \mu \mathrm{mol} / \mathrm{L}$, and thus, her estimated glomerular filtration rate (eGFR) was 35.4 $\mathrm{ml} / \mathrm{min} / 1.73 \mathrm{~m}^{2}$ according to the Chronic Kidney Disease Epidemiology Collaboration (CKD-EPI) Study formula. Considering that the decline in renal function was manifested more than $48 \mathrm{~h}$ after the use of $\mathrm{CM}$ and that the peak of $\mathrm{sCr}$ happened over a month after the operation, it is unlikely that the AKI of this patient was caused by CIN. We established rhabdomyolysis as the cause of AKI based on the abnormally high levels of serum myoglobin and CK. Rhabdomyolysis is a clinical syndrome characterized by injury to skeletal muscle fibers with disruption and release of their contents into the circulation. Myoglobin, CK and LDH are the most important substances for indicating muscle damage [14]. The clinical symptoms of rhabdomyolysis are well known: myalgia, weakness and swelling involving injured muscles, usually associated with the presence of myoglobin in urine (i.e. myoglobinuria). The clinical symptoms might include nonspecific symptoms such as fever, nausea, dyspepsia, and vomiting. Mild and subclinical cases of rhabdomyolysis, called in clinical practice myopathies, are typically characterized by elevated serum CK and myalgias [15]. The diagnosis of rhabdomyolysis is primarily based on myoglobinuria or a marked elevation in serum CK $[16,17]$. Rhabdomyolysis accounts for $13 \%-50 \%$ of AKI cases [18]. Its acquired causes include substance abuse, medication or toxic exposures, electrolyte abnormalities, endocrine disturbances, and autoimmune myopathies. Inherited predisposition to rhabdomyolysis can occur with disorders of glycogen metabolism, fatty acid $\beta$-oxidation, and mitochondrial oxidative phosphorylation [16]. The main driver of rhabdomyolysis induced kidney injury is myoglobin, which causes direct damage to renal proximal tubular cells, the formation of casts in distal tubules, renal vasoconstriction, and ischemia. Degradation of reabsorbed myoglobin in the proximal tubular cells leads to the elevation of ionized iron level, development of oxidative stress, and reduction of ATP synthesis. These metabolic changes further result in morphological and functional alterations such as development of acute tubular necrosis, which in concert with ischemia and obstructive damage of distal tubules can form a "vicious circle" [19-21]. Interestingly, this case was atypical because this patient had no definitive muscle pain and swelling. Nevertheless, in view of the tea-colored urine along with the results of laboratory examination, diagnosis can be defined as rhabdomyolysis. Regarding the absence of muscle swelling, hypotension and shock that are commonly associated with rhabdomyolysis, we do not exclude the possibility of a concurrent gastrointestinal tract infection in this patient.

The actual cause of rhabdomyolysis remains elusive. However, since this elderly patient received rosuvastatin for plaque stabilization after $\mathrm{PCl}$, it is possible that her rhabdomyolysis was induced by statin medication. Statins, also known as HMG-CoA reductase inhibitors, are frequently associated with mild muscle complaints. By contrast, their causing of rhabdomyolysis is extremely rare, probably only at a rate of 0.4 per 10,000 person per year [16]. Despite such a low incidence rate, certain drugs such as amiodarone that this patient has been taking to control ventricular rate could increase the risk of rhabdomyolysis when combined with statins [16]. In fact, a review of the United States Food and Drug Administration post-marketing database of rhabdomyolysis (CK 10,000) showed that, of 866 cases reported between 1987 and 2001, 44\% were related to combination therapy, as compared with statins alone [22]. The rosuvastatin specification mention that according to the FDA, the risk of myopathy during rosuvastatin therapy may be increased in Asian Americans because Asians appear to process the drug differently, half the standard dose can have the same cholesterol-lowering benefit in those patients, though a full dose could increase the risk of side-effects, which was indicated by the drug's manufacturer, AstraZeneca. Therefore, physicians should start Asian-American or East Asian patients at the lowest dose level. It also mention that for Asians, the starting dose is 5 $\mathrm{mg} / \mathrm{d}$, and for those who need to be more effective in reducing low-density lipoprotein cholesterol (LDL-C) in patients with 10 $\mathrm{mg} / \mathrm{d}$ can be considered as a starting dose, the dose can control the level of blood lipids in most patients, the maximum dose of this product is $20 \mathrm{mg} / \mathrm{d}$. All statins may be administered to patients with mild to moderate renal insufficiency; all require modified dosing for patients with substantial or severe renal disease [23]. The other study suggested that Rosuvastatin 2.5 mg had superior lipid lowering and cost effectiveness in dyslipidemic patients with concurrent CKD [24]. Moreover, rhabdomyolysis can occur as a result of electrolyte abnormalities (e.g. hypokalemia, hypophosphatemia, hyponatremia, and hypernatremia) in the setting of laxative and diuretic misuse/ abuse [25]. Collectively, the combination drug therapy and the presence of concurrent diarrhea and hypokalemia may increase the risk for this patient to develop rhabdomyolysis.

This patient developed serious agranulocytosis 7 days after treatment. Agranulocytosis is a blood dyscrasia characterized by a severe reduction of absolute neutrophil count (ANC) to less than 500 cells $/ \mu \mathrm{l}\left(0.5 \times 10^{9}\right.$ cells $\left./ L\right)$ in blood. The estimated incidence of agranulocytosis is 1.6 to 7.0 cases per million population per year $[26,27]$. Most cases of agranulocytosis are drug-induced, and its incidence appears to increase with age, particularly in elderly patients who receive, on average, more drugs than the younger patients [28]. The clinical manifestations of drug-induced agranulocytosis (DIA) include fever, septic shock, pneumonia, sore throat, cutaneous infection, and other infections [29]. The mechanism of DIA has not been fully elucidated; however, two main hypotheses have been proposed [30]. The immune mechanism is based on the presence of antibodies to circulating neutrophils found in the DIA patients, suggesting that an immunological reaction is occurring. Whereas the toxic mechanism proposes that the involvement of reactive metabolites produced by drug metabolism can stimulate a cascade of $\mathrm{T}$ cell-mediated reactions primarily against the myeloid cells [30,31]. All classes of medicines have been implicated in agranulocytosis. A systematic review has listed 125 drugs that are definitely or probably responsible for causing agranulocytosis. These drugs include analgesics, anticonvulsants, anti-infectives (including antibiotics, antiviral, and antifungal), antipsychotics, antithyroid drugs, antiplatelet agents, NSAIDs, as well as cardiovascular and gastrointestinal medications [32]. 
Many anti-infective drugs have been associated with idiosyncratic DIA. A monocentric study showed that antibiotics caused DIA in approximately $25 \%$ of the cases, with betalactams and trimethoprim-sulfamethoxazole being the most common rhabdomyolysis inducing antibiotics $[33,34]$.

In our case, the patient, an old lady without a history of blood and autoimmune diseases, developed agranulocytosis after a week of multidrug regimen that includes anti-infective (meropenem), antioxidant (GSH), and volume expansion agent (intralipid, Albumin). Despite only a few reports showing meropenem can cause agranulocytosis, we considered this is the case because her neutrophil count restored to normal when we withdrew meropenem and switched to G-CSF treatment. Thus, special care must be given to patients, especially elderly patients, who receive meropenem and a larger number of drugs at the same time, in order to prevent DIA.

\section{Conclusion}

Our case illustrates the importance of being cautious about the potential side effects and drug interactions in elderly people. In our case, both rhabdomyolysis and agranulocytosis are induced by drugs. Although these complications are uncommon, they are potentially fatal. Therefore, we need to pay more attention to the side effects in elderly patients, especially those taking a large number of drugs routinely.

\section{Acknowledgements}

This work is supported by Shanghai Pujiang Talent Plan (\#2016PJD032 to Y.L.) and research fund for returned overseas students from Ministry of Education (\#200550004 to Y.L.).

\section{Conflict of Interest}

The authors declare no conflict of interest.

\section{References}

1. Gurwitz JH (2004) Polypharmacy: a new paradigm for quality drug therapy in the elderly? Archives of internal medicine. 164: 1957-1959.

2. Kaufman DWKJP, Rosenberg L (2002) Recent patterns of medication use in the ambulatory adult population of the United States: the Slone survey. JAMA 287: 337-344.

3. Mehta RL, Kellum JA, Shah SV, Molitoris BA, Ronco C, et al. (2007) Acute Kidney Injury N. Acute Kidney Injury Network: report of an initiative to improve outcomes in acute kidney injury. Crit Care 11: R31.

4. Bellomo R, Ronco C, Kellum JA, Mehta RL, Palevsky P (2004) Acute Dialysis Quality Initiative w. Acute renal failure - definition, outcome measures, animal models, fluid therapy and information technology needs: the Second International Consensus Conference of the Acute Dialysis Quality Initiative (ADQI) Group. Crit Care 8: R204-212.

5. Susantitaphong P, Cruz DN, Cerda J, Abulfaraj M, Alqahtani F, et al. (2013) Acute Kidney Injury Advisory Group of the American Society of $\mathrm{N}$. World incidence of AKI: a meta-analysis. Clin J Am Soc Nephrol 8: 1482-1493.
6. Ftouh S, Lewington A (2014) Acute Kidney Injury Guideline Development Group convened by the National Clinical Guidelines $\mathrm{C}$, commissioned by the National Institute for $\mathrm{H}$, Care Excellence iawTRCoPC. Prevention, detection and management of acute kidney injury: concise guideline. Clin Med 14: 61-65.

7. Himmelfarb J (2009) Acute kidney injury in the elderly: problems and prospects. Semin Nephrol 29: 658-664.

8. Barretti P, Soares VA (1997) Acute renal failure: clinical outcome and causes of death. Ren Fail 19: 253-257.

9. Akcay A, Turkmen K, Lee D, Edelstein CL (2010) Update on the diagnosis and management of acute kidney injury. Int J Nephrol Renovasc Dis 3: 129-140.

10. Cartin-Ceba R, Kashiouris M, Plataki M, Kor DJ, Gajic O, et al (2012) Casey ET. Risk factors for development of acute kidney injury in critically ill patients: a systematic review and metaanalysis of observational studies. Crit Care Res Pract 2012: 691013.

11. Waikar SS, Liu KD, Chertow GM (2008) Diagnosis, epidemiology and outcomes of acute kidney injury. Clin J Am Soc Nephrol 3: 844-861.

12. Golshahi J, Nasri H, Gharipour M (2014) Contrast-induced nephropathy; A literature review. J Nephropathol 3: 51-56.

13. Stacul F, van der Molen AJ, Reimer P, Webb JA, Thomsen HS, et al (2011) Contrast Media Safety Committee of European Society of Urogenital R. Contrast induced nephropathy: updated ESUR Contrast Media Safety Committee guidelines. Eur Radiol 21: 2527-2541.

14. Bagley WH, Yang H, Shah KH (2007) Rhabdomyolysis. Intern Emerg Med 2: 210-218

15. Owczarek J, Jasinska M, Orszulak-Michalak D (2005) Drug-induced myopathies. An overview of the possible mechanisms. Pharmacol Rep 57: 23-34.

16. Nance JR, Mammen AL (2015) Diagnostic evaluation of rhabdomyolysis. Muscle Nerve 51: 793-810.

17. Melli G, Chaudhry V, Cornblath DR (2005) Rhabdomyolysis: an evaluation of 475 hospitalized patients. Medicine (Baltimore) 84: 377-385.

18. Zager RA (1989) Studies of mechanisms and protective maneuvers in myoglobinuric acute renal injury. Lab Invest 60: 619-629.

19. Ronco C, Bellomo R, Kellum JA (2009) Myoglobin as a toxin. Critical care nephrology 70: 1103-1109.

20. Zager RA (1996) Rhabdomyolysis and myohemoglobinuric acute renal failure. Kidney Int 49: 314-326.

21. Zager RA, Johnson AC, Becker K(2012) Plasma and urinary heme oxygenase-1 in AKI. J Am Soc Nephrol 23: 1048-1057.

22. Chang JT, Staffa JA, Parks M, Green L (2004) Rhabdomyolysis with HMG-CoA reductase inhibitors and gemfibrozil combination therapy. Pharmacoepidemiol Drug Saf 13: 417-426.

23. Agarwal R (2007) Effects of statins on renal function. Mayo Clinic proceedings 82 : 1381-1390.

24. Abe MMN, Maruyama T, Okada K, Soma M (2015) A Trial of Pitavastatin Versus Rosuvastatin for Dyslipidemia in Chronic Kidney Disease. J atheroscl thromb 22: 1235-1247.

25. Mas A, Chillaron JJ, Esteve E, Navalpotro I, Supervia A (2013) Severe rhabdomyolysis and hyponatremia induced by picosulfate and bisacodyl during the preparation of colonoscopy. Rev Esp Enferm Dig 105: 180-181. 
26. Andres E, Zimmer J, Affenberger S, Federici L, Alt M, et al. (2006) Idiosyncratic drug-induced agranulocytosis: Update of an old disorder. Eur J Intern Med 17: 529-535.

27. Kaufman DW (1991) The Drug etiology of agranulocytosis and aplastic anemia. New York, NY: Oxford University Press.

28. Andres E, Noel E, Kurtz JE, Henoun Loukili N, Kaltenbach G, et al. (2004) Life-threatening idiosyncratic drug-induced agranulocytosis in elderly patients. Drugs Aging 21: 427-435.

29. Pick AM, Nystrom KK (2014) Nonchemotherapy drug-induced neutropenia and agranulocytosis: could medications be the culprit? J Pharm Pract 27: 447-452.

30. Johnston A, Uetrecht $J$ (2015) Current understanding of the mechanisms of idiosyncratic drug-induced agranulocytosis. Expert Opin Drug Metab Toxicol 11: 243-257.
31. Tesfa D, Keisu M, Palmblad J (2009) Idiosyncratic drug-induced agranulocytosis: possible mechanisms and management. Am J Hematol 84: 428-434.

32. Andersohn F, Konzen C, Garbe E (2007) Systematic review: agranulocytosis induced by nonchemotherapy drugs. Ann Intern Med 146: 657-665.

33. Andres E, Maloisel F (2001) Antibiotic-induced agranulocytosis: a monocentric study of 21 cases. Arch Intern Med 161: 2619.

34. Andres E, Maloisel F, Kurtz JE, Kaltenbach G, Alt M, et al. (2002) Modern management of non-chemotherapy drug-induced agranulocytosis: a monocentric cohort study of 90 cases and review of the literature. Eur J Intern Med 13: 324-328. 